preparation. The adenoma detection rate $(A D R)$ was $62 \%$ with an mean of 2.1 polyps per colonoscopy in DM compared to ADR of $52 \%$ and mean 1.4 per colonoscopy in NDM. There was 1 cancer detected in each group. Neither group had any complications and no readmissions or 30 day mortality.

Conclusion Our findings were 3 fold. 1) Outcomes of Diabetic (DM) \& Non Diabetic (NDM) patients were similar for CIR, Cancers detected \& Adverse Events. 2) Bowel preparation is below $\mathrm{OA}$ \&GRS standards in DM patients which consequently led to significant number of repeat procedures and to failure to complete the colonoscopy in all of group of patients. 3) ADR and numbers found per colonoscopy seem to be greater in DM even with the poorer bowel prep. This may reflect the male preponderance but needs further investigation. We are looking at changing the bowel preparation from Moviprep to Kleen prep in the diabetic population within our programme.

Disclosure of Interest None Declared.

\section{PTH-057 A TWIN-CENTRE RANDOMIZED TRIAL OF INCREASING CLEAR FLUID INTAKE TO STANDARD MOVIPREP REGIMEN IN ADULT OUT-PATIENT COLONOSCOPY IMPROVES LEFT SIDED BOWEL CLEANSING \& POLYP DETECTION RATES}

doi:10.1136/gutjnl-2013-304907.544

1,"S Pelitari, ' J K Dowman, 'K Glover, 'S Keen, 'A D Farmer. 'Gastroenterology, Shrewsbury \& Telford NHS Trust, Telford, UK

Introduction Inadequate bowel preparation for colonoscopy is associated with increased complication rates and a reduction in diagnostic yield. Low volume $2 \mathrm{~L}$ polyethylene glycol and ascorbic acid (Moviprep, Norgine Pharmaceuticals) has been demonstrated to be non-inferior to other bowel cleansing agents but has greater patient tolerability (1). It is not known whether the addition of clear fluids to the standard regiment improves bowel cleansing with Moviprep.

Methods All adult patients attending for routine out-patient colonoscopy at two secondary care sites were randomised to receive either standard 2L Moviprep (regimen 1) or standard 2L Moviprep with an extra 1.5L of clear fluid (regimen 2). Segmental, and overall, bowel cleansing was assessed by the colonoscopist, blinded to the randomisation, using the validated Harefield Scale (HS). The HS is an inverted Ottawa scale scored from 4 (colon empty and clean) to 1 (large amounts of irremovable residual faeces) in six colonic segments.

Results 496 patients (252 females, mean age 59 years (range 22-90)) were included in an intention to treat analysis. The unadjusted completion rate was $95 \% .246$ patients were randomised to regimen 1 and 250 patients to regimen 2. Groups were similar in terms of demographics, indications for colonoscopy, presence of comorbidities and completion rates. Table 1 details the segmental, and overall, scoring between groups. Polyp detection rates were significantly higher in the group who took extra fluid (odds ratio 0.57 , $95 \%$ confidence interval $0.34-0.89, p=0.01$ ).

\section{Abstract PTH-057 Table 1}

\begin{tabular}{lccc}
\hline & $\begin{array}{c}\text { Regimen 1 (mean HS } \\
\text { score } \pm \text { SEM) }\end{array}$ & $\begin{array}{c}\text { Regimen 2 (mean HS } \\
\text { score } \pm \text { SEM) }\end{array}$ & $P$ value \\
\hline Rectum & $3 \pm 0.05$ & $3.5 \pm 0.05$ & $<0.0001$ \\
Sigmoid & $2.8 \pm 0.05$ & $3.3 \pm 0.03$ & $<0.0001$ \\
Descending colon & $2.7 \pm 0.06$ & $2.6 \pm 0.06$ & 0.3 \\
Transverse colon & $2.7 \pm 0.04$ & $2.6 \pm 0.07$ & 0.6 \\
Ascending colon & $2.6 \pm 0.05$ & $2.6 \pm 0.07$ & 0.8 \\
Caecum & $2.5 \pm 0.05$ & $2.6 \pm 0.1$ & 0.7 \\
Total score & $16.5 \pm 0.1$ & $17.3 \pm 0.01$ & 0.07 \\
\hline
\end{tabular}

Conclusion Increasing the volume of clear fluid intake with Moviprep improves cleansing in the distal colon and improves polyp detection rate. These data have important implications for clinical practise as upto $2 / 3$ rds of colorectal cancers arise in the left colon (2). Further research is now warranted to reproduce these findings in a larger cohort of patients.

Disclosure of Interest None Declared.

\section{REFERENCES}

1. Marmo et al. Effective bowel cleansing before colonoscopy: a randomised study of split-dosage versus non-split dosage regimens of high-volume versus low-volume polyethylene glycol solutions, GIE 2010; 72(2):313-320.

2. McCallion et al. Flexible sigmoidoscopy and the changing distribution of colorectal cancer: implications for screening, Gut 2001; 48(4):522-5

\section{PTH-058 TRAINEE COLONOSCOPISTS ACQUIRE COMPETENCY AT DIFFERENT RATES, AS DETERMINED BY CUSUM ANALYSIS OF COLONOSCOPY DATA FROM THE JETS DATABASE}

doi:10.1136/gutjnl-2013-304907.545

1,2, ${ }^{*} \mathrm{~S}$ T Ward, ${ }^{1} \mathrm{M}$ A Mohammed, ${ }^{2} \mathrm{~T}$ Ismail, ${ }^{3} \mathrm{R}$ Valori, ${ }^{3} \mathrm{P}$ Dunckley. ${ }^{1}$ University of Birmingham; '2Queen Elizabeth Hospital, Birmingham; ${ }^{3}$ Gloucestershire Hospitals NHS Trust, Gloucester, UK

Introduction The number of colonoscopies required to reach competency is not well established. Nevertheless, a minimal number forms part of UK certification criteria.

The Cusum technique is a statistical analysis of sequential data to determine if a process is 'in control'. The Joint Advisory Group on GI Endoscopy have developed an e-portfolio for users to record their endoscopic experience. The primary aim of this study was to determine the range of experience required by individuals to attain a caecal intubation rate (CIR) $\geq 90 \%$, as defined by Cusum. A secondary aim was to assess which training factors are associated with attaining competence.

Methods Inclusion criteria were all e-portfolio users who had performed $\leq 50$ ('baseline') colonoscopies prior to submission of data to the e-portfolio; termed 'trainees'. All colonoscopy records for the trainees were retrieved from the e-portfolio database and learning curve-Cusum analysis was performed. This analysis of colonoscopy completion reports the number of procedures required for CIR performance to reach $\geq 90 \%$. A colonoscopy was defined complete if the caecum or ileum was reached and was performed without assistance.

Trainees who had attained a CIR $\geq 90 \%$ were compared to those with a CIR $<90 \%$ for differences in previous endoscopic experience, case volume and other trainee factors by univariate (Mann-Whitney, Chi-squared) and multivariate (binomial logistic regression) analysis.

Results The e-portfolio contained 169,515 colonoscopy records entered by 1,572 different users. 265 users ('trainees') were confirmed to have performed $\leq 50$ baseline colonoscopies and were included in subsequent analyses. By Cusum method, 39 trainees attained a CIR $\geq 90 \% ; 226$ achieved a CIR $<90 \%$.

For those trainees with over 250 procedures, only $47 \%$ attained a CIR $\geq 90 \%$.

Factors associated with attaining $\mathrm{CIR} \geq 90 \%$ were high number of procedures $(\mathrm{P}<0.01)$, high number of colonoscopies per month $(\mathrm{P}<0.01)$, and prior experience of more than 100 sigmoidoscopies $(\mathrm{P}=0.017)$ by univariate and multivariate analysis. Nurse endoscopists attained competency at a higher rate than gastroenterology or surgical trainees by univariate $(\mathrm{P}=0.01)$ but not multivariate analysis. Conclusion This is the largest study to date by both procedure and trainee numbers assessing colonoscopy competency by Cusum method.

Trainees achieve competency at different rates. A high proportion of trainees will not attain a CIR $>90 \%$ even after 250 procedures. 
High case volume and prior sigmoidoscopy experience are associated with a CIR $>90 \%$. The potential of both these factors to influence the attainment of competency should be exploited within endoscopy training programmes.

Disclosure of Interest None Declared.

\section{PTH-059 UPPER GASTROINTESTINAL MALIGNANCIES: 6976 ENDOSCOPY REVIEW IN A MULTINATIONAL STUDY}

doi:10.1136/gutjnl-2013-304907.546

${ }_{1,}^{1,}$ S Roy. 'Gastroenterology, Queen Mary's Hospital, Sidcup, UK

Introduction To compare the prevalence of upper gastrointestinal (UGI) malignant and premalignant conditions in three separate populations; British, Japanese and Arabian.

Methods 6976 Upper Gastrointestinal (UGI) endoscopies were retrospectively reviewed in a multinational comparative study. This involved three population groups: Group A- British \& others $(\mathrm{n}=2158)$; Group B- Japanese $(\mathrm{n}=2628)$; Group C- Arabians (Saudi Arabians \& others $(n=2190))$.

The majority of the patients fell in the above 16 age group.

The patients presented with UGI symptoms and were selected at random. The data was collected between 1986 and 2012

The study involved patients from Barnsley District General Hospital (Barnsley), Darent Valley Hospital (Dartford) \& Queen Mary's Hospital (Sidcup) in UK - Group A; Showa University Fujigaoka Hospital \& Niigata Cancer Centre Hospital (Japan)- Group B; Jubail Hospitals (Saudi Arabia) - Group C.

Results A comparison was made for the malignant and premalignant diseases. It revealed a very high incidence of UGI malignancies amongst Japanese (Group B). There is significant number of malignant diseases amongst non-Arabians, but such malignancies amongst Arabians are rare.

The incidence of gastric ulcers and gastric polyps are very high amongst Japanese compared to the other population groups in the study.

It is found that the prevalence of Barrett's Oesophagus has increased significantly in the last ten years.

The results are summarised as below:

UGI malignancies recorded:

Group A: British population: 37 (1.71\% with 95\% CI: 1.17 to 2.26) 6.51)

Group B: Japanese population: 148 (5.63\% with 95\% CI: 4.75 to

Group C: Arabian population: 16 (1.17\% with 95\% CI: 1.17 to 2.26)

Ulcers in the upper GI tract recorded:

Group A: British population: 366 (16.96\%, 95\% CI: 15.38 to 18.54)

Group B: Japanese population: 498 (18.95\% 95\% CI: 17.45 to 20.45)

Group C: Saudi population: $506(23.11 \%, 95 \%$ CI $=21.34$ to 24.87).

Conclusion It can be concluded that Arabians (Saudis) suffer rarely from UGI malignancies and it may be related to social, environmental, geographical, genetic and dietary habits. Dietary habits in Japan are very different from the British and Saudi population.

Alcohol consumption amongst Saudis is very little. In comparison, Japanese and UK populations drink alcohol significantly more.

Prevalence of Barrett's has increased significantly in the last 10 years. It is likely due to effective Helicobacter Pylori eradication and due to increasing awareness of Barrett's amongst endoscopists.

Disclosure of Interest None Declared.

\section{PTH-060 ACUTE UPPER GASTROINTESTINAL BLEEDING TO BE ENDOSCOPED WITHIN 24 HOURS OF ADMISSION - ARE WE MEETING BSG AND NICE GUIDELINES? THE EXPERIENCE OF A DISTRICT GENERAL HOSPITAL}

doi:10.1136/gutjnl-2013-304907.547

1J Barbour, ${ }^{1, *}$ S Smith, ${ }^{1} \mathrm{~K}$ St-Louis. 'Gateshead NHS Trust, Gateshead, UK

Introduction BSG and NICE guidelines recommend that all patients with a suspected GI bleed have an endoscopy within 24 hours of admission. The Queen Elizabeth Hospital (OEH), Gateshead (a District General Hospital serving a population of approximately 200,000) provides an urgent out of hours GI bleed service. Additionally there is an 8.30am slot in endoscopy from Monday to Friday allocated to patients who have symptoms of an upper GI bleed. We audited our compliance to the 24 hour guideline.

Methods The electronic reporting system 'Endosoft' was searched using the terms: Upper GI bleed; Malaena; or Haematemesis, for all referrals for gastroscopy between January $1^{\text {st }} 2011$ and December 31st 2011. The date, time of endoscopy, findings and interventions were noted. Time of admission, theatre use and In-patient and 4 week mortality were also noted.

Results 162 patients were admitted with acute GI bleeding over the 12 month period. Overall 126 (78\%) were endoscoped within 24 hours. 35 of the 36 patients endoscoped in theatre had their procedure within 24 hours of admission. 16 (44\%) of the 36 patients outside the 24 hour window were admitted on a Friday evening or Saturday. Of the 36 patients that suffered a delay to endoscopy: 5 had oesophageal varices; 1 a gastric varix; 6 peptic ulcer disease (4 high risk stigmata and 2 low risk); 2 Mallory Weiss tear, and 22 were normal. Of the 36 patients who went straight to theatre, 34 (94\%) had an endoscopic cause for bleeding identified. $20(56 \%)$ had variceal bleeds, 12 had peptic ulcer disease (10 high risk stigmata and 2 low risk). Overall in-patient mortality of those found to have an upper GI cause for bleeding was $16 \%$ (15 of 91 ) and 4 week mortality was 18\% (17 of 91). Five (29\%) of those that died were cirrhotic patients and $8(47 \%)$ had peptic ulcer disease with high risk stigmata. 3 of the 17 deaths occurred in the patients who were delayed prior to endoscopy, one of whom came in at a time when there was no allocated 8.30am slot the following morning. All 3 of the mortalities who waited over 24 hours for their procedure had endoscopic intervention. There was however no significant difference (chi-square test) between mortality of the delayed patients and those scoped within $24 \mathrm{hrs}$ (8\% compared to $11 \%$ ).

Conclusion During 2011 the $\mathrm{OEH}$ appropriately identified sick patients suitable for the out of hours GI bleed service, with $94 \%$ of theatre cases requiring endoscopic intervention. The NICE and BSG guidelines of endoscoping patients referred for a GI bleed within 24 hours of admission was met in $78 \%$ of cases. Allocated $8.30 \mathrm{am}$ endoscopy slots on Saturday and Sunday would improve these figures, but it is currently unclear if this would affect the patient outcome.

Disclosure of Interest None Declared.

\section{PTH-061 TO EVALUATE THE SAFETY AND OUTCOMES OF ESD AND HYBRID ESD FOR LARGE SESSILE COLORECTAL POLYPS INCLUDING PROCEDURAL COMPLICATIONS AND RECURRENCE RATE}

doi:10.1136/gutjnl-2013-304907.548

1.*S M Ali, 'N Radhakrishnan, 'R Hammonds, 'R George. 'Gastroenterology, Pennine Acute Hospitals NHS Trust, Manchester, UK

Introduction The purpose of ESD and Hybrid ESD (circumferential excision and snaring) is to obtain en bloc specimen. Margins are checked for residual tissue and APC applied if appropriate 